\begin{tabular}{|c|l|}
\hline Title & Linear dependence of certain L-values of half-integral weight modular forms \\
\hline Author(s) & Katsurada, Hidenori; Mizuno, Yoshinori \\
\hline Citation & Hokkaido University Preprint Series in Mathematics, 944, 1-21 \\
\hline Issue Date & 2009-820 \\
\hline DOI & 10.14943/84091 \\
\hline Doc URL & http://hdl.handle.net/2115/69751 \\
\hline Type & bulletin (article) \\
\hline File Information & pre944.pdf \\
\hline
\end{tabular}

Instructions for use 


\title{
Linear dependence of certain L-values of half-integral weight modular forms
}

\author{
Hidenori Katsurada and Yoshinori Mizuno*
}

2009 July 1

\section{Introduction}

For two holomorphic modular forms

$$
h_{1}(z)=\sum_{m=0}^{\infty} c_{1}(m) \mathbf{e}(m z) \text { and } h_{2}(z)=\sum_{m=0}^{\infty} c_{2}(m) \mathbf{e}(m z)
$$

of half-integral weights $k_{1}-1 / 2$ and $k_{2}-1 / 2$, respectively, of Neben Typus for $\Gamma_{0}(N)$, we define the convolution product $L\left(s, h_{1}, h_{2}\right)$ as

$$
L\left(s, h_{1}, h_{2}\right)=L_{N}\left(2 s-k_{1}-k_{2}+3, \omega\right) \sum_{m=1}^{\infty} \frac{c_{1}(m) c_{2}(m)}{m^{s}}
$$

where $\omega$ is a certain Dirichlet character determined by the Neben characters of $h_{1}$ and $h_{2}$, and

$$
L_{N}(s, \omega)=\sum_{d>0,(d, N)=1} \frac{\omega(d)}{d^{s}}
$$

(for the precise definition of $\omega$, see Section 2.) The analytic properties of this Dirichlet series were investigated by Shimura [12] (see also Mizuno, [10].) Furthermore the algebraicity of the values of this Dirichlet serries evaluated at halfintegers was deeply investigated by Shimura [12]. However, as far as we know, there is no result about the algebraicity of its special values at integers. In this paper, we investigate the linear dependence of the special values of the RankinSelberg convolution products at integers of two half-integral weight modular forms. To state our main result, let $h$ be a cusp form of half-integral weight

*The second named author was partly supported by Grant-in-Aid for JSPS Fellows for this work. 
$k-1 / 2$ for $\Gamma_{0}(4)$ belonging to Kohnen's plus subspace, and $E_{3 / 2}^{(\chi)}$ the twisted Zagier Eisenstein series by a Dirichlet character $\chi$ (for the precsie definition of $E_{3 / 2}^{(\chi)}$, see Section 2.) Let $N=p_{1}^{e_{1}} \cdots p_{r}^{e_{r}}$ be the prime decomposition of an odd integer $N$, and put $\tilde{N}=p_{1} \cdots p_{r}$. For a primitive character $\chi$ of conductor $N$, we define $\mathcal{E}_{3 / 2}^{(\chi)}(z)$ by

$$
\mathcal{E}_{3 / 2}^{(\chi)}(z)=\sum_{0<M \mid \tilde{N}} E_{3 / 2}^{\left(\left(\frac{*}{M}\right) \chi\right)}(z),
$$

where $\left(\frac{*}{M}\right)$ denotes the Jacobi symbol. Assume that $\left(\frac{*}{M}\right) \chi$ is primitive for any positive divisor $M$ of $\tilde{N}$. Then $\mathcal{E}_{3 / 2}^{(\chi)}(z)$ is a (non-holomorphic) modular form of weight $3 / 2$ and character $\chi^{2}$ for $\Gamma_{0}\left(4 N^{2}\right)$. Then our main result (Theorem 4.2) can be stated as follows:

Under the above notation and the assumption, there exsits a positive integer $r$ depending only on $h$ such that the values $L\left(l, h, \mathcal{E}_{3 / 2}^{\left(\chi_{1}\right)}\right), \ldots, L\left(l, h, \mathcal{E}_{3 / 2}^{\left(\chi_{r+1}\right)}\right)$ are linearly dependent over $\overline{\mathbf{Q}}$ for any integer $1 \leq l \leq k-2$ and Dirichlet characters $\chi_{1}, \ldots, \chi_{r+1}$ of odd conductors such that $\chi_{1}^{2}, \ldots, \chi_{r}^{2}, \chi_{r+1}^{2}$ are primitive. In particular, the values $L\left(l, h, \mathcal{E}_{3 / 2}^{\left(\chi_{1}\right)}\right), \ldots, L\left(l, h, \mathcal{E}_{3 / 2}^{\left(\chi_{r+1}\right)}\right)$ are linearly dependent over $\overline{\mathbf{Q}}$ for any non-quadratic characters $\chi_{1}, \ldots, \chi_{r+1}$ of odd prime conductors.

We note that there is no non-trivial linear relation among the functions $L\left(s, h, \mathcal{E}_{3 / 2}^{\left(\chi_{1}\right)}\right), \ldots, L\left(s, h, \mathcal{E}_{3 / 2}^{\left(\chi_{r}\right)}\right)$ and $L\left(s, h, \mathcal{E}_{3 / 2}^{\left(\chi_{r+1}\right)}\right)$ themselves in general (cf. Remark after Theorem 4.2.) At present we don't know whether this type of result holds for any two holomorphic modular forms of half-integral weight. Nevetheless this result seems a little bit surprising. We hope the above result shed a new right on this subject.

A main tool for proving Theorem 4.2 is the twisted Koecher-Maaß series. Let $F(Z)$ be a modular form of weight $k$ with respect to the symplectic group $S p_{n}(\mathbf{Z})$. Then $F(Z)$ has the following Fourier expansion:

$$
F(Z)=\sum_{A} c_{F}(A) \exp (2 \pi \sqrt{-1} \operatorname{tr}(A Z))
$$

where $A$ runs over all semi-positive definite half-integral matrices over $\mathbf{Z}$ of degree $n$ and $\operatorname{tr}(X)$ denotes the trace of a matrix $X$. For a positive integer $N$ let $S L_{n, N}(\mathbf{Z})=\left\{U \in S L_{n}(\mathbf{Z}) \mid U \equiv 1_{n} \bmod N\right\}$, and $\epsilon_{N}(T)=\#\{U \in$ $\left.S L_{n, N}(\mathbf{Z}) \mid T[U]=T\right\}$. For a primitive Dirichlet character $\chi \bmod N$ we define the Koecher-Maaß series $L(s, F, \chi)$ of $F$ twisted by $\chi$ as

$$
L(s, F, \chi)=\sum_{T} \frac{\chi(\operatorname{tr}(T)) c_{F}(T)}{\epsilon_{N}(T)(\operatorname{det} T)^{s}},
$$


where $T$ runs over a complete set of representatives of $S L_{n, N}(\mathbf{Z})$-equivalence classes of positive definite half-integral matrices of degree $n$. We note that this Dirichlet series coincides with the Hecke $L$-function associated to $F$ twisted by $\chi$ in case $n=1$. Though we are mainly concerned with $L(s, F, \chi)$ in this paper, we also define another type of the twisted Koecher-Maaß series $L^{*}(s, F, \chi)$ as

$$
L^{*}(s, F, \chi)=\sum_{T} \frac{\chi(\operatorname{det}(2 T)) c_{F}(T)}{\epsilon(T)(\operatorname{det} T)^{s}},
$$

where $T$ runs over a complete set of representatives of $S L_{n}(\mathbf{Z})$-equivalence classes of positive definite half-integral matrices of degree $n$, and $\epsilon(T)=\epsilon_{1}(T)$. These two Dirichlet series $L(s, F, \chi)$ and $L^{*}(s, F, \chi)$ coincide with each other in case $n=1$, but they don't in general. To distinguish these two Dirichlet series, we sometimes call $L(s, F, \chi)$ and $L^{*}(s, F, \chi)$ the twisted Koecher-Maaß series of the first and second kind, respectively. We will discuss the relation between these two Dirichlet series in case $n=2$ in Section 3. We write $L(s, F)=L\left(s, F, \chi_{0}\right)$ if $\chi_{0}$ is the principal character. The analytic and arithmetic properties of the Koecher-Maaß series $L(s, F, \chi)$ were investigated by Choie and Kohnen [3] (cf. Propositions 1.1 and 1.2.) In particular, its special values at integers have been studied in a general setting by them. In this paper we give an explicit formula of $L(s, M(h), \chi)$ for the Maaß lift $M(h)$ of a cusp form $h$ in the Kohnen plus subspace of weight $k-1 / 2$ for $\Gamma_{0}(4)$ and apply it to the special values of convolution products of the half integral weight modular forms. To be more precise, we express $L(s, M(h), \chi)$ in terms of Rankin-Selberg convolution products of $h$ and the twisted Zagier Eisenstein series of weight $3 / 2$ (cf. Theorem 2.3.) This also gives a certain generalization of Böcherer's result in [2]. To prove Theorem 2.3, for a modular form $F$ with respect to $S p_{2}(\mathbf{Z})$ we express $L(s, F, \chi)$ in terms of the sum of twisted Koecher-Maaß series of the second kind (cf. Theorem 3.8.) On the other hand, we easily get an explicit formula for the twisted Koecher-Maaß series of the second kind for the Maaß lift (cf. Proposition 2.1.) Thus we get Theorem 2.3. By using Theorem 2.3 combined Proposition 1.2, we can immediately get Theorem 4.2.

Furthermore, by using Theorem 2.3, we get the algebraicity of the special values of $L(s, M(h), \chi)$ at half-integers for the Hecke eigenform $h$ of half-integral weight stated above. To explain this more precisely, let $S(h)$ be the normalized Hecke eigenform corresponding to $h$ under the Shimura correspondence, and $\mathbf{Q}(S(h))$ the field generated over $\mathbf{Q}$ by all the Fourier coefficients of $f$. As is well known, $\mathbf{Q}(S(h))$ is a totally real field. Furthermore, let $u_{-}(S(h))$ be the complex numbers defined in [12]. Then under a certain condition, the value $L(l / 2, M(h), \chi)$ belongs to the vector space $\mathbf{Q}(S(h)) u_{-}(S(h))(\chi) \pi^{l-1} \sqrt{-1}$ for any odd integer such that $1 \leq l \leq 2 k-3$ and a primitive Dirichlet character $\chi$ of odd conductor (cf. Theorem 4.1.) This type of result was obtained for the Ikeda lift in [7] when $\chi$ is the principal character. On the other hand, in this 
paper, we get such a result for infinitely many primitive character. We note that the above theorem is not a special case of Choie and Kohnen's result. Actually they treated the values of Koecher-Maaß series at integers. On the other hand, we treat the values at half-integers. Finally we also get a functional equation of the Rankin-Selberg convolution product stated above (cf. Theorem 4.4.)

Notation. We denote by $\mathbf{e}(x)=\exp (2 \pi \sqrt{-1} x)$ for a complex number $x$. For a commutative ring $R$, we denote by $M_{m n}(R)$ the set of $(m, n)$-matrices with entries in $R$. For an $(m, n)$-matrix $X$ and an $(m, m)$-matrix $A$, we write $A[X]={ }^{t} X A X$, where ${ }^{t} X$ denotes the transpose of $X$. Let $a$ be an element of $R$. Then for an element $X$ of $M_{m n}(R)$ we often use the same symbol $X$ to denote the $\operatorname{coset} X \bmod a M_{m n}(R)$. Put $G L_{m}(R)=\left\{A \in M_{m}(R) \mid \operatorname{det} A \in R^{*}\right\}$, where $\operatorname{det} A$ denotes the determinant of a square matrix $A$, and $R^{*}$ denotes the unit group of $R$. Let $S_{n}(R)$ denote the set of symmetric matrices of degree $n$ with entries in $R$. Furthermore, for an integral domain $R$ of characteristic different from 2 , let $\mathcal{L}_{n}(R)$ denote the set of half-integral matrices of degree $n$ over $R$, that is, $\mathcal{L}_{n}(R)$ is the set of symmetric matrices of degree $n$ whose $(i, j)$-component belongs to $R$ or $\frac{1}{2} R$ according as $i=j$ or not. We call an element of $2 \mathcal{L}_{n}(R)$ an even-integral matrix over $R$. We simply write $\mathcal{L}_{n}(\mathbf{Z})$ as $\mathcal{L}_{n}$. In particular, if $S$ is a subset of $S_{n}(\mathbf{R})$ with $\mathbf{R}$ the field of real numbers, we denote by $S_{>0}$ (resp. $S_{\geq 0}$ ) the subset of $S$ consisting of positive definite (resp. semi-positive definite) matrices. $G L_{n}(R)$ acts on the set $S_{n}(R)$ in the following way:

$$
G L_{n}(R) \times S_{n}(R) \ni(g, A) \longrightarrow{ }^{t} g A g \in S_{n}(R)
$$

Let $G$ be a subgroup of $G L_{n}(R)$. For a subset $\mathcal{B}$ of $S_{n}(R)$ stable under the action of $G$ we denote by $\mathcal{B} / G$ the set of equivalence classes of $\mathcal{B}$ with respect to $G L_{n}(R)$. We sometimes identify $\mathcal{B} / G$ with a complete set of representatives of $\mathcal{B} / G$. We abbreviate $\mathcal{B} / G L_{n}(R)$ as $\mathcal{B} / \sim$ if there is no fear of confusion. Two symmetric matrices $A$ and $A^{\prime}$ with entries in $R$ are called equivalent with each other with respect to $G$ and write $A \tilde{G}_{G} A^{\prime}$ if there is an element $X$ of $G$ such that $A^{\prime}=A[X]$. We also write $A \sim A^{\prime}$ if there is no fear of confusion. For square matrices $X$ and $Y$ we write $X \perp Y=\left(\begin{array}{cc}X & O \\ O & Y\end{array}\right)$.

\section{Twisted Koecher-Maaß series}

We denote by $\mathbf{H}_{n}$ the Siegel's upper half space of degree $n$. Let $\Gamma_{n}$ be the symplectic group of degree $n$. For a positive integer $k$, we denote by $\mathfrak{M}_{k}\left(\Gamma_{n}\right)$ (resp. $\mathfrak{\Im}_{k}\left(\Gamma_{n}\right)$ the space of holomorphic modular forms (resp. cusp forms) of weight $k$ for $\Gamma_{n}$. For a modular form $F(Z)$ of weight $k$ with respect to $\Gamma_{n}$ and a Dirichlet character $\chi \bmod N$ let $L(s, F, \chi)$ be the Koecher-Maaß series for $F$ twisted by 
$\chi$ as in the Introduction. We review the analytic and arithmetic properties of $L(s, F, \chi)$ following Choie and Kohnen [3] (see also Maaß [9], Arakawa [1].)

Proposition 1.1. Let $F \in \mathfrak{\Xi}_{k}\left(\Gamma_{n}\right)$, and $\chi$ a primitive character of conductor N. Put

$$
\gamma_{n}(s)=(2 \pi)^{-n s} \prod_{i=1}^{n} \pi^{i-1} / 2 \Gamma(s-(i-1) / 2),
$$

and put

$$
\Lambda(s, F, \chi)=N^{2 s} \tau(\chi)^{-1} L(s, F, \chi) \quad(\operatorname{Re}(s)>>0),
$$

where $\tau(\chi)$ is the Gauss sum of $\chi$. Then $\Lambda(s, F, \chi)$ has an analytic continuation to the whole s-plane and has the following functional equation:

$$
\Lambda(k-s, F, \chi)=(-1)^{n k / 2} \chi(-1) \Lambda(s, F, \bar{\chi}) .
$$

Proposition 1.2 Let $F \in \mathbb{\Xi}_{k}\left(\Gamma_{n}\right)$. Then there exists a $\mathbf{Z}$-module $M_{f} \subset \mathbf{C}$ of finite rank such that

$$
\frac{N L(m, F, \chi)}{\tau(\chi)(2 \pi \sqrt{-1})^{n m}} \in M_{f} \otimes_{\mathbf{Z}} \mathbf{Z}[\chi]
$$

for any primitive character $\chi$ of conductor $N$ and any integer $m$ such that $(n+$ 1) $/ 2 \leq m \leq k-(n+1) / 2$, where $\mathbf{Z}[\chi]$ is the $\mathbf{Z}$-module obtained from by adjoining the values of $\chi$.

We remark that the analytic and the arithmetic properties of $L^{*}(s, F, \chi)$ are not so well-known in general.

\section{Twisted Koecher-Maaß series of the Maaß lift}

From now on we assume that $k$ is an even positive integer. Let $N$ be a positive integer, and $\chi$ be a Dirichlet character $\bmod N$. We then denote by $\mathfrak{R}_{k-1 / 2}^{\infty}\left(\Gamma_{0}(4 N), \chi\right)$ the space of $C^{\infty}$ modular forms of weight $k-1 / 2$ and Neben character $\chi$ for $\Gamma_{0}(4 N)$. Furthermore we denote by $\mathfrak{R}_{k-1 / 2}\left(\Gamma_{0}(4 N), \chi\right)\left(\operatorname{resp} . \mathfrak{S}_{k-1 / 2}\left(\Gamma_{0}(4 N), \chi\right)\right.$ the space of holomorphic modular forms (resp. cusp forms) of weight $k-1 / 2$ and Neben character $\chi$ for $\Gamma_{0}(4 N)$. We write $\mathfrak{R}_{k-1 / 2}^{\infty}\left(\Gamma_{0}(4 N)\right)$ and the others if $\chi$ is a trivial character. We denote by $\mathfrak{R}_{k-1 / 2}^{+}\left(\Gamma_{0}(4 N)\right)\left(\operatorname{resp} . \mathfrak{S}_{k-1 / 2}^{+}\left(\Gamma_{0}(4 N)\right)\right)$ Kohnen's plus subspace of $\mathfrak{R}_{k-1 / 2}\left(\Gamma_{0}(4 N)\right.$ ) (resp. $\mathfrak{\subseteq}_{k-1 / 2}\left(\Gamma_{0}(4 N)\right)$ ). As for the precise definition of the Kohnen plus subspace, see [8]. Let $h$ be an element of $\mathfrak{\Im}_{k-1 / 2}\left(\Gamma_{0}(4)\right)$. Then $h$ has the following Fourier expansion:

$$
h(z)=\sum_{e} c(e) \mathbf{e}(e z),
$$


where $e$ runs over all positive integers such that $(-1)^{k-1 / 2} e \equiv 0,1 \bmod 4$. For $T=\left(\begin{array}{cc}n & r / 2 \\ r / 2 & m\end{array}\right) \in \mathcal{L}_{2>0}$, put

$$
A(T)=\sum_{d \mid G . C . D(n, r, m)} d^{k-1} c\left(\frac{4 n m-r^{2}}{d^{2}}\right)
$$

and

$$
M(h)(Z)=\sum_{T \in \mathcal{L}_{2}>0} A(T) \mathbf{e}(\operatorname{tr}(T Z)) .
$$

Then $M(h)$ is a cusp form of weight $k$ for $\Gamma_{2}$. Furthermore if $h$ is a Hecke eigenform, so is $M(h)$, and the spinor L-function $L(s, M(h)$, Sp) can be expressed as

$$
L(s, M(h), \mathrm{Sp})=\zeta(s-k+1) \zeta(s-k+2) L(s, S(h)),
$$

where $S(h)$ is the Hecke eigenform in $S_{2 k-2}\left(\Gamma_{1}\right)$ corresponding to $h$ under the Shimura correspondence (cf. [5].) We call $M(h)$ the Maaß lift of $h$. For any $D \in \mathbf{Z}_{>0}$, we put

$$
H_{1}(D)=\sum_{A \in \mathcal{L}_{2>0}(D) / S L_{2}(\mathbf{z})} \frac{1}{\epsilon(A)},
$$

where $\mathcal{L}_{2>0}(D)=\left\{A \in \mathcal{L}_{2>0} \mid 4 \operatorname{det} A=D\right\}$, and $\epsilon(A)=\#\left\{U \in S L_{2}(\mathbf{Z}) \mid A[U]=\right.$ $A\}$. Furthermore, for a Dirichlet character $\eta$ of conductor $N$, we define Zagier's Eisenstein series $E_{3 / 2}^{(\eta)}(z)$ of weight $3 / 2$ twisted by $\eta$ as

$$
E_{3 / 2}^{(\eta)}(z)=\sum_{m=0}^{\infty} 2 \eta(m) H_{1}(m) \mathbf{e}(m z)+y^{-1 / 2} \sum_{n=-\infty}^{\infty} \beta\left(4 \pi n^{2} y\right) \mathbf{e}\left(-n^{2} z\right) \eta\left(-n^{2}\right),
$$

where $z=x+\sqrt{-1} y, H_{1}(0)=\frac{-1}{24}$ and

$$
\beta(x)=\frac{1}{16 \pi} \int_{1}^{\infty} u^{-3 / 2} e^{-x u} d u .
$$

If $\eta$ is primitive, then $E_{3 / 2}^{(\eta)}(z)$ belongs to $\mathfrak{R}_{3 / 2}^{\infty}\left(\Gamma_{0}\left(4 N^{2}\right), \eta^{2}\right)$, and there exists a constants $A, a, b>0$ such that $\left|E_{3 / 2}^{(\eta)}(z)\right| \leq A\left(y^{a}+y^{-b}\right)$ for any $z=x+\sqrt{-1} y \in$ $\mathbf{H}_{1}$. Let $N=p_{1}^{e_{1}} \cdots p_{r}^{e_{r}}$ be the prime decomposition of $N$, and put $\tilde{N}=p_{1} \cdots p_{r}$. We then define $\mathcal{E}_{3 / 2}^{(\eta)}(z)$ by

$$
\mathcal{E}_{3 / 2}^{(\eta)}(z)=\sum_{0<M \mid \tilde{N}} E_{3 / 2}^{\left(\left(\frac{*}{M}\right) \eta\right)}(z)
$$

where $\left(\frac{*}{M}\right)$ denotes the Jacobi symbol. Assume that $\left(\frac{*}{M}\right) \eta$ is primitive for any positive divisor $M$ of $\tilde{N}$. Then $\mathcal{E}_{3 / 2}^{(\eta)}(z)$ belongs to $\mathfrak{R}_{3 / 2}^{\infty}\left(\Gamma_{0}\left(4 N^{2}\right), \eta^{2}\right)$. Furthermore 
assume that $p \equiv-1 \bmod 4$ for some prime factor $p$ of $N$. Then $\mathcal{E}_{3 / 2}^{(\eta)}(z)$ is holomorphic on $\mathbf{H}_{1}$ and therefore by the above growth condition, we see that it belongs to $\left.\mathfrak{R}_{3 / 2}\left(\Gamma_{0}\left(4 N^{2}\right), \eta^{2}\right)\right)$. Now let $k$ and $l$ be positive integers and $\eta_{1}$ and $\eta_{2}$ Dirichlet character mod $N_{1}$ and $N_{2}$, respepctively. For $h_{1}(z)=\sum_{m=0}^{\infty} c_{1}(m) \mathbf{e}(m z) \in$ $\mathfrak{R}_{k-1 / 2}\left(\Gamma_{0}\left(N_{1}\right), \eta_{1}\right)$, and an element $h_{2}(z)$ of $\mathfrak{R}_{l-1 / 2}^{\infty}\left(\Gamma_{0}\left(N_{2}\right), \eta_{2}\right)$ with the following Fourier expansion

$$
h_{2}(z)=\sum_{m=0}^{\infty} c_{2}(m)(m z)+y^{-1 / 2} \sum_{n=-\infty}^{\infty} b(n, y) \mathbf{e}\left(-n^{2} z\right)
$$

we define the convolution product $L\left(s, h_{1}, h_{2}\right)$ as

$$
L\left(s, h_{1}, h_{2}\right)=L_{N}(2 s-k-l+3, \omega) \sum_{m=1}^{\infty} \frac{c_{1}(m) c_{2}(m)}{m^{s}}
$$

as in Section 0, where $\omega(d)=\left(\frac{-1}{d}\right)^{k-l} \eta_{1}(d) \eta_{2}(d)$, and

$$
L_{N}(s)=\sum_{m>0,(m, N)=1} \frac{\omega(d)}{d^{s}} .
$$

If $\chi$ is a primitive Dirichlet character $\bmod N$, and $h$ belongs to $\mathfrak{R}_{k-1 / 2}\left(\Gamma_{0}(4)\right)$, then we can also define $L\left(s, h, E_{3 / 2}^{(\chi)}\right)$ regarding $h$ as an element of $\mathfrak{M}_{k-1 / 2}\left(\Gamma_{0}\left(4 N^{2}\right)\right)$. Now let $N$ be a positive integer, and $N=p^{e_{1}} \cdots p^{e_{r}}$ be the prime decomposition of $N$. Let $\chi$ be a Dirichlet character $\bmod N$. Fix a prime factor $p$ of $N$. For an integer $n$ prime to $p$, take an integer $m$ such that

$$
m \equiv\left\{\begin{array}{c}
n \bmod p^{e} \\
1 \bmod N / p^{e}
\end{array} .\right.
$$

We then put

$$
\chi^{(p)}(n)=\left\{\begin{array}{cl}
\chi(m), & \text { if }(n, p)=1 \\
0, & \text { if }(n, p) \neq 1
\end{array} .\right.
$$

Then it is independent of the choice of $m$, and $\chi^{(p)}$ is a character $\bmod p^{e}$. Furthermore we have

$$
\chi=\prod_{p \mid N} \chi^{(p)} .
$$

First we give an explicit formula of $L^{*}(s, M(h), \chi)$.

Proposition 2.1. Let

$$
h(z)=\sum_{e} c(e) \mathbf{e}(e z)
$$


be an element of $\mathfrak{S}_{k-1 / 2}^{+}\left(\Gamma_{0}(4)\right)$, and $M(h)$ be the Maaß lift of $h$ to $\mathfrak{\Xi}_{k}\left(\Gamma_{2}\right)$. Then for a primitive character $\chi$ we have

$$
L^{*}(s, M(h), \chi)=2^{2 s-1} L\left(s, h, E_{3 / 2}^{(\chi)}\right) .
$$

Proof. This is a natural generalization of the result given in Satz 3 [2] p.20. For $T=\left(\begin{array}{cc}a_{11} & a_{12} / 2 \\ a_{12} / 2 & a_{22}\end{array}\right) \in \mathcal{L}_{2}$, put $e(T)=G . C . D\left(a_{11}, a_{12}, a_{22}\right)$, and $A(T)$ the $T$-th Fourier coefficient of $M(h)$. Then, collecting the terms by the value $e=e(T)$, one has

$$
\begin{aligned}
L^{*}(s, M(h), \chi) & =\sum_{e=1}^{\infty} \sum_{T_{0} \in L_{2>0} / S L_{2, N}(\mathbf{Z}), e\left(T_{0}\right)=1} \frac{\chi\left(\operatorname{det}\left(2 e T_{0}\right)\right) A\left(e T_{0}\right)}{\epsilon\left(e T_{0}\right)\left(\operatorname{det} e T_{0}\right)^{s}} \\
& =\sum_{e=1}^{\infty} \frac{\chi^{2}(e)}{e^{2 s}} \sum_{T_{0} \in L_{2>0} / S L_{2, N}(\mathbf{Z}), e\left(T_{0}\right)=1} \frac{\chi\left(\operatorname{det}\left(2 T_{0}\right)\right) A\left(e T_{0}\right)}{\epsilon\left(T_{0}\right)\left(\operatorname{det} T_{0}\right)^{s}} .
\end{aligned}
$$

By the definition of the Fourier coefficient $A(T)$ of $M(h)$, we see that the inner sum is equal to

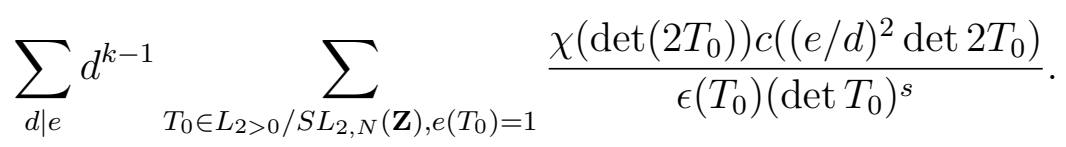

Thus we obtain

$$
\begin{aligned}
L^{*}(s, M(h), \chi) & =\sum_{m=1}^{\infty} \frac{\chi^{2}(m)}{m^{2 s-k+1}} \sum_{n=1}^{\infty} \frac{\chi(n)}{n^{2 s}} \sum_{T_{0} \in L_{2>0} / S L_{2}(\mathbf{Z}), e\left(T_{0}\right)=1} \frac{\chi\left(\operatorname{det}\left(2 T_{0}\right)\right) c\left(n^{2} \operatorname{det} 2 T_{0}\right)}{\epsilon\left(T_{0}\right)\left(\operatorname{det} T_{0}\right)^{s}} \\
& =L\left(2 s-k+1, \chi^{2}\right) \sum_{T \in L_{2}>0 / S L_{2}(\mathbf{Z})} \frac{\chi(\operatorname{det}(2 T)) c(\operatorname{det}(2 T))}{\epsilon(T)(\operatorname{det} T)^{s}}
\end{aligned}
$$

Collecting the terms in the summation by the value $d=\operatorname{det} 2 T$, we have

$$
L^{*}(s, M(h), \chi)=2^{2 s} L\left(2 s-k+1, \chi^{2}\right) \sum_{d=1}^{\infty} \frac{c(d) \chi(d) H_{1}(d)}{d^{s}} .
$$

This proves the assertion.

Our calculations are also applicable to the Siegel-Eisenstein series of degree 2 and even weight $k \geq 4$ defined by

$$
E_{2, k}(Z)=\sum_{\{C, D\}} \operatorname{det}(C Z+D)^{-k}, \quad Z \in \mathbf{H}_{2},
$$


where the sum is taken over all non-associated coprime symmetric pairs $\{C, D\}$ of degree 2. For a non-negative integer $m$, the Cohen function $H(k-1, m)$ is given by $H(k-1, m)=L_{-m}(2-k)$. Here

$$
\begin{aligned}
L_{D}(s) & \left\{\begin{aligned}
\zeta(2 s-1), & D=0 \\
L\left(s, \chi_{D_{K}}\right) \sum_{a \mid f} \mu(a) \chi_{D_{K}}(a) a^{-s} \sigma_{1-2 s}(f / a), & D \neq 0, D \equiv 0,1 \bmod 4 \\
0, & D \equiv 2,3 \bmod 4,
\end{aligned}\right.
\end{aligned}
$$

where the positive integer $f$ is defined by $D=D_{K} f^{2}$ with the discriminant $D_{K}$ of $K=\mathbf{Q}(\sqrt{D}), \chi_{D_{K}}$ is the Kronecker symbol, $\mu$ is the Möbius function and $\sigma_{s}(n)=\sum_{d \mid n} d^{s}$. For $k \geq 4$, the Cohen Eisenstein series $\mathcal{H}_{k-1}(z)$ is

$$
\mathcal{H}_{k-1}(z)=\sum_{m=0}^{\infty} H(k-1, m) \mathbf{e}(m z) .
$$

It is known that $\mathcal{H}_{k-1}(z)$ is a modular form of weight $k-1 / 2$ belonging to the Kohnen plus space and that the Saito-Kurokawa lift of $\mathcal{H}_{k-1}(z)$ coincides with $E_{2, k}(Z)$ up to a scalar multiple, namely the $T$-th Fourier coefficient of $E_{2, k}(Z)$ for a positive definite $T$ is ( $B_{k}$ : the $k$-th Bernoulli number)

$$
\frac{4 k(k-1)}{B_{k} B_{2 k-2}} \sum_{d \mid e(T)} d^{k-1} H\left(k-1, \frac{\operatorname{det} 2 T}{d^{2}}\right) .
$$

By the same argument as above, we have

Proposition 2.2. Let $N$ and $\chi$ be as in Proposition 2.1. Then we have

$$
L^{*}\left(s, E_{2, k}, \chi\right)=2^{2 s+2} \frac{k(k-1)}{B_{k} B_{2 k-2}} L\left(s, \mathcal{H}_{k-1}, E_{3 / 2}^{(\chi)}\right)
$$

Now a main tool for the proof of Theorem 4.2 is:

Theorem. 2.3. Let $h$ be a Hecke eigenform in $\mathfrak{S}_{k-1 / 2}^{+}\left(\Gamma_{0}(4)\right)$. Let $N$ be an odd positive integer, and $N=p_{1}^{e_{1}} \cdots p_{r}^{e_{r}}$ be the prime decomposition of $N$. Let $\chi$ be a primitive Dirichlet character $\bmod N$.

(1). If $\chi^{\left(p_{i}\right)}(-1)=-1$ for some $i$. Then we have $L(s, M(h), \chi)=0$.

(2). Assume that $\chi^{\left(p_{i}\right)}(-1)=1$ for any $i$. Fix a character $\tilde{\chi}$ such that $\tilde{\chi}^{2}=\chi$. Then we have

$$
\left.L(s, M(h), \chi)=2^{2 s} \prod_{i=1}^{r}\left(1-\left(\frac{-1}{p_{i}}\right) p_{i}^{-1}\right) N^{2}\left(\frac{-1}{N}\right) L\left(s, h, \mathcal{E}_{3 / 2}^{(\tilde{\chi})}\right)\right) .
$$


The above theorem is not so easy to prove as Proposition 2.1, and we prove it in the next section. Similarly we have

Theorem. 2.4. Let $N$ and $\chi$ be as in Theorem 2.3.

(1). If $\chi^{\left(p_{i}\right)}(-1)=-1$ for some $i$. Then we have $L\left(s, E_{2, k}, \chi\right)=0$.

(2). Assume that $\chi^{\left(p_{i}\right)}(-1)=1$ for any $i$. Fix a character $\tilde{\chi}$ such that $\tilde{\chi}^{2}=\chi$. Then we have

$$
\left.L\left(s, E_{2, k}, \chi\right)=2^{2 s+2} \frac{k(k-1)}{B_{k} B_{2 k-2}} \prod_{i=1}^{r}\left(1-\left(\frac{-1}{p_{i}}\right) p_{i}^{-1}\right) N^{2}\left(\frac{-1}{N}\right) L\left(s, \mathcal{H}_{k-1}, \mathcal{E}_{3 / 2}^{(\tilde{\chi})}\right)\right) .
$$

Remark 1. The expression in (2) of the above two theorems does not depend on the choice of $\tilde{\chi}$.

Remark 2. In [6], and [7], the first named author got an explicit formula for $L(s, F)$ when $F$ is the Klingen Eisenstein lift and the Ikeda lift of an elliptic cuspidal Hecke eigenform, respectively. It is interesting to generalize these results to twisted cases.

\section{Twisted Koecher-Maaß series of degree two}

Let $\chi$ be a primitive character $\bmod N$. For $A \in \mathcal{L}_{n>0}$, put

$$
h(A, \chi)=\sum_{\bar{U} \in S L_{n}(\mathbf{Z} / N \mathbf{Z})} \chi(\operatorname{tr}(A[U])) .
$$

Proposition 3.1. Let

$$
F(Z)=\sum_{A \in \mathcal{L}_{n \geq 0}} c_{F}(A) \mathbf{e}(\operatorname{tr}(A Z))
$$

be an element of $\mathfrak{M}_{k}\left(\Gamma_{n}\right)$. Assume $N \neq 2$. Then we have

$$
L(s, F, \chi)=\sum_{A \in \mathcal{L}_{n>0} / S L_{n}(\mathbf{Z})} \frac{c_{F}(A) h(A, \chi)}{\epsilon(A)(\operatorname{det} A)^{s}} .
$$

Proof. Put $\mathcal{L}_{A}=\left\{A^{\prime} \in \mathcal{L}_{N>0} \mid A^{\prime} \sim_{S L_{n}(\mathbf{Z})} A\right\}$. Then we have

$$
L(s, F, \chi)=\sum_{A} \frac{c_{F}(A)}{(\operatorname{det} A)^{s}} \sum_{A^{\prime} \in \mathcal{L}_{A} / S L_{n, N}(\mathbf{Z})} \frac{\chi\left(\operatorname{tr}\left(A^{\prime}\right)\right)}{\epsilon_{N}\left(A^{\prime}\right)} .
$$


Fix an element $A$ of $\mathcal{L}_{N>0}$. Let $U_{1}$ and $U_{2}$ be elements of $S L_{n}(\mathbf{Z})$. Then $A\left[U_{1}\right]$ and $A\left[U_{2}\right]$ are $S L_{n, N}(\mathbf{Z})$-equivalent with each other if $U_{2} \in S O_{n}(A) S L_{n, N}(\mathbf{Z}) U_{1}$, where $S O_{n}(A)=\left\{U \in S L_{n}(\mathbf{Z}) \mid A[U]=A\right\}$. Thus we can take $\{A[U] \mid U \in$ $\left.S O_{n}(A) S L_{n, N}(\mathbf{Z}) \backslash S L_{n}(\mathbf{Z})\right\}$ as a complete set of representatives of $\mathcal{L}_{A} / S L_{n, N}(\mathbf{Z})$. Furthermore we have $\operatorname{tr}\left(A\left[U_{1}\right]\right)=\operatorname{tr}\left(A\left[U_{2}\right]\right)$ if $U_{2}=S O_{n}(A) S L_{n, N}(\mathbf{Z}) U_{1}$. Furthermore we have $\epsilon_{N}\left(A^{\prime}\right)=1$ for any $A^{\prime} \in \mathcal{L}_{n>0}$. Thus we have

$$
\begin{gathered}
\epsilon(A) \sum_{A^{\prime} \in \mathcal{L}_{A} / S L_{n, N}(\mathbf{Z})} \frac{\chi\left(\operatorname{tr}\left(A^{\prime}\right)\right)}{\epsilon_{N}\left(A^{\prime}\right)}=\epsilon(A) \sum_{U \in S O_{n}(A) S L_{n, N}(\mathbf{Z}) \backslash S L_{n}(\mathbf{Z})} \chi(\operatorname{tr}(A[U])) \\
=\sum_{U \in S L_{n, N}(\mathbf{Z}) \backslash S L_{n}(\mathbf{Z})} \chi(\operatorname{tr}(A[U])) .
\end{gathered}
$$

This proves the assertion.

Now we shall compute $h(A, \chi)$ in the case where $A$ is an element of $\mathcal{L}_{2>0}$. First we remark the following elementary lemma, which can be proved by Chinese remainder theorem.

Lemma 3.2 . Let $A \in \mathcal{L}_{n>0}$. Let $N$ be an odd positive integer, and $N=$ $p^{e_{1}} \cdots p^{e_{r}}$ be the prime decomposition of $N$. Let $\chi$ be a primitive Dirichlet character $\bmod N$. Then we have

$$
h(A, \chi)=\prod_{i=1}^{r} h\left(A, \chi^{\left(p_{i}\right)}\right) .
$$

From now on let $A$ be an element of $\mathcal{L}_{2>0}$. For each $c \in \mathbf{Z}$, put

$$
\begin{gathered}
R_{N}(A, c)=\left\{\mathbf{x}=\left(x_{1}, x_{2}, x_{3}, x_{4}\right) \in(\mathbf{Z} / N \mathbf{Z})^{4} \mid(A \perp A)[\mathbf{x}]-c \equiv 0 \bmod N\right. \\
\text { and } \left.x_{1} x_{4}-x_{2} x_{3}-1 \equiv 0 \bmod N\right\} .
\end{gathered}
$$

Then we have

$$
h(A, \chi)=\sum_{c=1}^{N} \chi(c) \#\left(R_{N}(A, c)\right) .
$$

To compute $\# R_{N}(A, c)$, we need two lemmas. The following lemma can easily be proved by using the usual $p$-adic Newton approximation method.

Lemma 3.3. Let $A$ be as above. Let $p$ be a prime number. Assume that $\left(\frac{4 \operatorname{det} A}{p}\right)=-1$ or $\left(\frac{4 \operatorname{det} A}{p}\right)=0$. Then we have

$$
\# R_{p^{e}}(A, c)=p^{2(e-1)} \# R_{p}(A, c)
$$


for any $c \in \mathbf{Z}$ prime to $p$.

We define the Gauss sum $\tau_{p}$ by

$$
\tau_{p}=\sum_{r=1}^{p}\left(\frac{r}{p}\right) \mathbf{e}(r / p)
$$

as usual. Then the following lemma is well known.

Lemma 3.4. Let $p$ be a prime number, and e a positive integer. For any $u \in \mathbf{Z}$ prime to $p$ we have

$$
\sum_{x=1}^{p^{e}} \mathbf{e}\left(u x^{2}\right)=p^{e / 2} \text { or }\left(\frac{u}{p}\right) p^{(e-1) / 2} \tau_{p}
$$

according as e is even or odd.

Corollary. We have

$$
\sum_{\left(x_{1}, x_{2}\right) \in\left(\mathbf{Z} / p^{e} \mathbf{Z}\right)^{2}} \mathbf{e}\left(u_{1} x_{1}^{2}+u_{2} x_{2}^{2}\right)=p^{e}\left(\frac{-1}{p}\right)^{e} .
$$

Proposition 3.5. Let $p$ be an odd prime number. Let $A$ be a symmetric matrix of degree 2 with entries in $\mathbf{Z}_{p}$. Assume that $A \not \equiv O \bmod p$, and that $\left(\frac{4 \operatorname{det} A}{p}\right)=-1$ or $\left(\frac{4 \operatorname{det} A}{p}\right)=0$ Then for any $c \in F_{p}^{\times}$, we have

$$
\# R_{p^{e}}(A, c)=p^{2(e-1)}\left(p-\left(\frac{-1}{p}\right)\right)\left(p-\left(\frac{-4 \operatorname{det} A}{p}\right)\right) .
$$

Proof. We may assume that $A=\left(\begin{array}{ll}a & 0 \\ 0 & d\end{array}\right)$ with $a \neq 0$. For $s, t \in F_{p}$ put

$$
I(A, c ; s, t)=\sum_{\left(x_{1}, x_{2}, x_{3}, x_{4}\right) \in F_{p}^{4}} \mathbf{e}\left(\left(a\left(x_{1}^{2}+x_{2}^{2}\right)+d\left(x_{3}^{2}+x_{4}^{2}\right)-c\right) s+\left(x_{1} x_{4}-x_{2} x_{3}-1\right) t\right),
$$

and

$$
I(A, c)=\sum_{(s, t) \in F_{p}^{2}} I(A, c ; s, t)
$$

Then we have

$$
I(A, c)=p^{2} \# R_{p}(A, c) .
$$


It is easily shown that we have

$$
I(A, c ; 0, t)=p \#\left\{\left(x_{1}, x_{2}, x_{3}, x_{4}\right) \in F_{p}^{4} \mid x_{1} x_{4}-x_{2} x_{3}=1\right\}=p^{4}-p^{2} .
$$

We note that

$$
I(A, c ; s, t)=\sum_{\left(y_{1}, y_{2}, y_{3}, y_{4}\right) \in F_{p}^{4}} \mathbf{e}\left(a s\left(y_{1}^{2}+y_{2}^{2}\right)+a s\left(4 a d s^{2}-t^{2}\right)\left(y_{3}^{2}+y_{4}^{2}\right)-s c-t\right) .
$$

Thus, by Corollary to Lemma 3.4, for any $s \in F_{p}^{\times}$, we have

$$
I(A, c ; s, t)=p\left(\frac{-1}{p}\right) \sum_{\left(y_{3}, y_{4}\right) \in F_{p}^{2}} \mathbf{e}\left(a s\left(4 a d s^{2}-t^{2}\right)\left(y_{3}^{2}+y_{4}^{2}\right)-s c-t\right) .
$$

Then for any $s \in F_{p}^{\times}$and $t \in F_{p}^{\times}$we have $4 a d s^{2}-t^{2} \in F_{p}^{\times}$. Thus we have

$$
I(A, c ; s, t)=\left(p\left(\frac{-1}{p}\right)\right)^{2} \mathbf{e}(-c s-t)=p^{2} \mathbf{e}(-c s-t) .
$$

First assume that $\left(\frac{4 \operatorname{det} A}{p}\right)=-1$. Then for any $s \in F_{p}^{\times}$we have $4 a d s^{2} \in F_{p}^{\times}$. Thus we have

$$
I(A, c ; s, 0)=\left(p\left(\frac{-1}{p}\right)\right)^{2} \mathbf{e}(-c s)=p^{2} \mathbf{e}(-c s) .
$$

Thus we have

$$
\sum_{s \in F_{p}^{\times}, t \in F_{p}} I(A, c ; s, t)=0
$$

and therefore we have

$$
I(A, c)=p^{4}-p^{2}
$$

This implies that we have

$$
\# R_{p}(A, c)=p^{2}-1
$$

Next assume that $\left(\frac{4 \operatorname{det} A}{p}\right)=0$. Then $d=0$. Thus for any $s \in F_{p}^{\times}$we have

$$
I(A, c ; s, 0)=p^{3}\left(\frac{-1}{p}\right) \mathbf{e}(-c s) .
$$

Thus we have

$$
\begin{gathered}
\sum_{s \in F_{p}^{\times}, t \in F_{p}} I(A, c ; s, t)=p^{2} \sum_{s \in F_{p}^{\times}, t \in F_{p}^{\times}} \mathbf{e}(-c s-t)+p^{3}\left(\frac{-1}{p}\right) \sum_{s \in F_{p}^{\times}} \mathbf{e}(-c s) \\
=p^{2}-p^{3}\left(\frac{-1}{p}\right),
\end{gathered}
$$


and therefore we have

$$
I(A, c)=p^{4}-p^{3}\left(\frac{-1}{p}\right) .
$$

This implies that we have

$$
\# R_{p}(A, c)=p\left(p-\left(\frac{-1}{p}\right)\right) .
$$

This proves the assertion for $e=1$, and by Lemma 3.3, we prove the assertion for a general case.

Proposition 3.6. Let $A$ be as in Proposition 3.7. Assume that $\left(\frac{4 \operatorname{det} A}{p}\right)=1$. Then for any $c \in \mathbf{Z}$ let $r=\operatorname{ord}_{p}\left(4 \operatorname{det} A-c^{2}\right)$. Then we have

$$
\# R_{p}(A, c)=p^{2 e-2}\left(p-\left(\frac{-1}{p}\right)\right)\left(p \sum_{i=e-r}^{e}\left(\frac{-1}{p}\right)^{e-i}-\sum_{i=e-r-1}^{e-1}\left(\frac{-1}{p}\right)^{e-i}\right)
$$

if $r \leq e-1$, and

$$
\# R_{p}(A, c)=p^{2 e-2}\left(p-\left(\frac{-1}{p}\right)\right)\left(p \sum_{i=0}^{e}\left(\frac{-1}{p}\right)^{e-i}-\sum_{i=0}^{e-1}\left(\frac{-1}{p}\right)^{e-i}\right)
$$

if $r=e$.

Remark. The above assertion for $e=1$ can also be proved by using the same argument as Proposition 3.5. However, we have to prove the general case in another way because Lemma 3.4 does not hold in this case.

Proof. We may assume that $A=\left(\begin{array}{ll}a & 0 \\ 0 & d\end{array}\right)$. Let $l$ be an integer such that $l^{2} \equiv 4 a d \bmod p^{e}$. Put

$\tilde{R}_{p^{e}}(A, c)=\left\{\left(y_{1}, y_{2}, y_{3}, y_{4}\right) \in\left(\mathbf{Z} / p^{e} \mathbf{Z}\right)^{4} \mid y_{1}^{2}+y_{2}^{2}+y_{3}^{2}+y_{4}^{2}-2 a c=y_{1}^{2}-y_{2}^{2}+y_{3}^{2}-y_{4}^{2}-2 a l=0\right\}$.

Then the linear transformation $2 a x_{1}=y_{1}+y_{4}, 2 a x_{2}=y_{2}-y_{3}, 2 l x_{3}=l\left(y_{2}+\right.$ $\left.y_{3}\right), 2 l x_{4}=y_{1}-y_{4}$ induces a bijection from $R_{p^{e}}(A, c)$ to $\tilde{R}_{p^{e}}(A, c)$. Put

$$
=\sum_{(s, t) \in\left(\mathbf{Z} / p^{e} \mathbf{Z}\right)^{2}} \sum_{\left(y_{1}, y_{2}, y_{3}, y_{4}\right) \in\left(\mathbf{Z} / p^{e} \mathbf{Z}\right)^{4}} \mathbf{e}\left(\left(s\left(y_{1}^{2}+y_{2}^{2}+y_{3}^{2}+y_{4}^{2}-2 a c\right)+t\left(y_{1}^{2}-y_{2}^{2}+y_{3}^{2}-y_{4}^{2}-2 a l\right)\right) / p^{e}\right) .
$$

Then we have

$$
I(A, c)=p^{2 e} \# \tilde{R}_{p^{e}}(A, c) .
$$


We have

$$
=\sum_{(u, v) \in\left(\mathbf{Z} / p^{e} \mathbf{Z}\right)^{2}} \sum_{\left(y_{1}, y_{2}, y_{3}, y_{4}\right) \in\left(\mathbf{Z} / p^{e} \mathbf{Z}\right)^{4}} \mathbf{e}\left(\left(u\left(y_{1}^{2}+y_{3}^{2}\right)+v\left(y_{2}^{2}+y_{4}^{2}\right)-a(c+l) u+a(-c+l) v\right) / p^{e}\right) .
$$

We remark that $c+l \not \equiv 0 \bmod p$, or $c-l \not \equiv 0 \bmod p$. First assume that $c-l \not \equiv 0 \bmod p$, and put $-a(c+l)=p^{r} \alpha$, and $a(-c+l)=\beta$ with $\alpha, \beta \not \equiv 0 \bmod p$. For each $(u, v) \in\left(\mathbf{Z} / p^{e} \mathbf{Z}\right)^{2}$ put

$$
I(A, c ; u, v)=\sum_{\left(y_{1}, y_{2}, y_{3}, y_{4}\right) \in\left(\mathbf{Z} / p^{e} \mathbf{Z}\right)^{4}} \mathbf{e}\left(\left(u\left(y_{1}^{2}+y_{3}^{2}\right)+v\left(y_{2}^{2}+y_{4}^{2}\right)+p^{r} \alpha u+\beta v\right) / p^{e}\right),
$$

and for $i, j=1, \ldots, e$

$$
I^{(i, j)}(A, c)=\sum_{\substack{u, v) \in(\mathbf{Z} / p e \mathbf{Z})^{2} \\ \text { ord } p(u)=i, \operatorname{ord}_{p}(v)=j}} I(A, c ; u, v) .
$$

Then we have

$$
I(A, c)=\sum_{i, j=1}^{e} I^{(i, j)}(A, c)
$$

We note that for any $i=1, \ldots, e$ and $1 \leq j \leq e-2$, we have

$$
I^{(i, j)}(A, c)=0
$$

We have

$$
\begin{gathered}
I^{(i, e)}(A, c)=p^{2 e} \sum_{\substack{u \in \mathbf{Z} / p^{e} \mathbf{Z} \\
\text { ord } p(u)=i}} \sum_{\left(y_{1}, y_{3}\right) \in\left(\mathbf{Z} / p^{e} \mathbf{Z}\right)^{2}} \mathbf{e}\left(\left(u\left(y_{1}^{2}+y_{3}^{2}\right)+p^{r} \alpha u\right) / p^{e}\right) \\
=p^{2 e} \sum_{u \in\left(\mathbf{Z} / p^{e-i} \mathbf{Z}\right)^{\times}} \sum_{\left(y_{1}, y_{3}\right) \in\left(\mathbf{Z} / p^{e} \mathbf{Z}\right)^{2}} \mathbf{e}\left(\left(u\left(y_{1}^{2}+y_{3}^{2}\right)+p^{r} \alpha u\right) / p^{e-i}\right) \\
=p^{2 e+2 i} \sum_{u \in\left(\mathbf{Z} / p^{e-i} \mathbf{Z}\right)^{\times}} \sum_{\left(y_{1}, y_{3}\right) \in\left(\mathbf{Z} / p^{e-i} \mathbf{Z}\right)^{2}} \mathbf{e}\left(\left(u\left(y_{1}^{2}+y_{3}^{2}\right)+p^{r} \alpha u\right) / p^{e-i}\right) \\
=p^{2 e+2 i} p^{e-i}\left(\frac{-1}{p}\right)^{e-i} \sum_{u \in\left(\mathbf{Z} / p^{e-i} \mathbf{Z}\right)^{\times}} \mathbf{e}\left(\alpha u / p^{e-r-i}\right) .
\end{gathered}
$$

For $1 \leq i \leq e-1$ we have

$$
\sum_{u \in\left(\mathbf{Z} / p^{e-i} \mathbf{Z}\right)^{\times}} \mathbf{e}\left(\alpha u / p^{e-r-i}\right)=0,-1, \text { or } p^{e-i-r-1}(p-1)
$$


according as $e-i-r \geq 2, e-i-r=-1$, or $e-i-r \leq 0$. Furthermore we have $I^{(e, e)}(A, c)=p^{4 e}$. Thus we have

$$
\sum_{i=1}^{e} I^{(i, e)}(A, c)=p^{4 e} \sum_{i=e-r}^{e}\left(\frac{-1}{p}\right)^{e-i}-p^{4 e-1} \sum_{i=e-r-1}^{e-1}\left(\frac{-1}{p}\right)^{e-i}
$$

or

$$
p^{4 e} \sum_{i=0}^{e}\left(\frac{-1}{p}\right)^{e-i}-p^{4 e-1} \sum_{i=0}^{e-1}\left(\frac{-1}{p}\right)^{e-i}
$$

according as $r \leq e-1$ or $r=e$. Similarly we have

$$
\sum_{i=1}^{e} I^{(i, e-1)}(A, c)=-p^{4 e-1}\left(\frac{-1}{p}\right) \sum_{i=e-r}^{e}\left(\frac{-1}{p}\right)^{e-i}+p^{4 e-2}\left(\frac{-1}{p}\right) \sum_{i=e-r-1}^{e-1}\left(\frac{-1}{p}\right)^{e-i}
$$

or

$$
-p^{4 e-1}\left(\frac{-1}{p}\right) \sum_{i=0}^{e}\left(\frac{-1}{p}\right)^{e-i}+p^{4 e-2}\left(\frac{-1}{p}\right) \sum_{i=0}^{e-1}\left(\frac{-1}{p}\right)^{e-i}
$$

according as $r \leq e-1$ or $r=e$. This proves the assertion.

Proposition 3.7. Let $N$ be an odd positive integer, and $N=p^{e_{1}} \cdots p^{e_{r}}$ be the prime decomposition of $N$. Let $\chi$ be a primitive Dirichlet character mod $N$. Let $\chi^{\left(p_{i}\right)}$ be the primitive Dirichlet character $\bmod p^{e_{i}}$ such that $\chi=\chi^{\left(p_{1}\right)} \cdots \chi^{\left(p_{r}\right)}$.

(1) Assume that $\chi^{\left(p_{i}\right)}(-1)=-1$ for some $i$. Then we have

$$
h(A, \chi)=0 .
$$

(2) Assume that $\chi^{\left(p_{i}\right)}(-1)=1$ for any $i$. Then we have

$$
\left.h(A, \chi)=\prod_{i=1}^{r}\left\{\left(1+\left(\frac{4 \operatorname{det} A}{p_{i}}\right)\right)\left(1-\left(\frac{-1}{p_{i}}\right) p_{i}^{-1}\right)\right\} N^{2}\left(\frac{-1}{N}\right) \tilde{\chi}(4 \operatorname{det} A)\right),
$$

where $\tilde{\chi}$ is a character such that $\tilde{\chi}^{2}=\chi$.

Proof. (1) Assume that $\chi^{\left(p_{i}\right)}(-1)=-1$ for some $i$. Take integers $a, b$ such that $a^{2}+b^{2} \equiv-1 \bmod p^{e_{i}}$, and put $\tilde{U}_{0}=\left(\begin{array}{cc}-b & a \\ a & b\end{array}\right)$. Then we have $\operatorname{det} \tilde{U}_{0} \equiv$ $1 \bmod p^{e_{i}}$, and ${ }^{t} \tilde{U}_{0} \tilde{U}_{0} \equiv-1_{2} \bmod p^{e_{i}}$. Thus there exists an element $U_{0} \in S L_{2}(\mathbf{Z})$ such that ${ }^{t} U_{0} U_{0} \equiv-1_{2} \bmod p^{e_{i}}$. Then for any $B=\left(\begin{array}{cc}b_{11} & b_{12} / 2 \\ b_{12} / 2 & b_{22}\end{array}\right)$ we have

$$
\operatorname{tr}\left(B\left[U_{0}\right]\right)=b_{11} b^{2}-b_{12} a b+b_{22} a^{2}+b_{11} a^{2}-b_{12} b a+b_{22} a^{2} \equiv-\operatorname{tr}(B) \bmod p^{e_{i}}
$$


Thus we have

$$
\begin{gathered}
h(A, \chi)=\sum_{\bar{U} \in S L_{2}(\mathbf{Z} / p \mathbf{Z})} \chi\left(\operatorname{tr}\left(A\left[U U_{0}\right]\right)\right. \\
=\sum_{\bar{U} \in S L_{2}(\mathbf{Z} / p \mathbf{Z})} \chi(-\operatorname{tr}(A[U])=\chi(-1) h(A ; \chi) .
\end{gathered}
$$

This proves (1).

(2) By Lemma 3.3, it suffices to prove the case $N=p^{e}$ with $p$ a prime number. We recall that we have

$$
h(A, \chi)=\sum_{c \in \mathbf{Z} / p^{e} \mathbf{Z}} \chi(c) \# R_{p^{e}}(A ; c) .
$$

First assume that $\left(\frac{4 \operatorname{det} A}{p}\right)=-1$. Then the number $\# R_{p^{e}}(A ; c)$ does not depend on $c$. Thus we have

$$
\sum_{c \in \mathbf{Z} / p_{i}^{e} \mathbf{Z}} \chi(c) \# R_{p^{e}}(A ; c)=0
$$

Next assume that $\left(\frac{4 \operatorname{det} A}{p}\right)=1$. For $r \leq e$ put

$$
S_{r, A}=\left\{x \in \mathbf{Z} / p^{e} \mathbf{Z} \mid x^{2} \equiv 4 \operatorname{det} A \bmod p^{r}\right\} .
$$

Then by Proposition 2,7 we have

$$
\begin{gathered}
h(A, \chi)=\sum_{c \in \mathbf{Z} / p^{e} \mathbf{Z}} \chi(c) \# R_{p^{e}}(A ; c) \\
=\sum_{r=0}^{e-1} \sum_{u \in S_{r, A}} \chi(u) p^{2 e-2}\left(p-\left(\frac{-1}{p}\right)\right)\left(p\left(\frac{-1}{p}\right)^{r}-\left(\frac{-1}{p}\right)^{r+1}\right)+\sum_{u \in S_{e, A}} \chi(u) p^{2 e-1}\left(p-\left(\frac{-1}{p}\right)\right)\left(\frac{-1}{p}\right)^{e} .
\end{gathered}
$$

Since $\chi$ is a primitive character, we have

$$
\sum_{u \in S_{r, A}} \chi(u)=0
$$

for any $r \leq e-1$. Thus we have

$$
h(A, \chi)=2 p^{2 e-1}\left(p-\left(\frac{-1}{p}\right)\right)\left(\frac{-1}{p}\right)^{e} \chi(l),
$$

where $l$ is an integer such that $l^{2} \equiv 4 \operatorname{det} A \bmod p^{e}$. Now there exists a character $\tilde{\chi}$ such that $\tilde{\chi}^{2}=\chi$. Then we have $\chi(l)=\tilde{\chi}(4 \operatorname{det} A)$, and this value $\operatorname{does}$ not depend on the choice of $\tilde{\chi}$. This proves the assertion (2).

Now by Proposition 3.7, we immediately derive the following. 
Theorem 3.8. Let $F$ be an element of $\mathfrak{M}_{k}\left(\Gamma_{n}\right)$. Let $N$ be an odd positive integer, and $N=p_{1}^{e_{1}} \cdots p_{r}^{e_{r}}$ be the prime decomposition of $N$. Let $\chi$ be a primitive Dirichlet character mod $N$.

(1). If $\chi^{\left(p_{i}\right)}(-1)=-1$ for some $i$. Then we have $L(s, F, \chi)=0$.

(2). Assume that $\chi^{\left(p_{i}\right)}(-1)=1$ for any $i$. Fix a character $\tilde{\chi}$ such that $\tilde{\chi}^{2}=\chi$. Then we have

$$
L(s, F, \chi)=\prod_{i=1}^{r}\left(1-\left(\frac{-1}{p_{i}}\right) p_{i}^{-1}\right) N^{2}\left(\frac{-1}{N}\right) \sum_{M \mid \tilde{N}} L^{*}\left(s, F,\left(\frac{*}{M}\right) \tilde{\chi}\right),
$$

where $\tilde{N}=p_{1} \cdots p_{r}$.

Proof of Theorems 2.3 and 2.4. The assertions can be directly proved by Propositions 2.1, 2.2, and Theorem 3.8.

\section{Special values of convolution products of half- integral weight modular forms and twisted Koecher-Maaß series}

For a holomorphic modular form $g$ of integral or half-integral weight, we denote by $\mathbf{Q}(g)$ the field generated over $\mathbf{Q}$ by all the Fourier coefficients of $g$. First we recall the following result due to Shimura [11], [12] under more general setting.

Proposition 4.1. (1) Let $f$ be a Hecke eigenform in $\mathfrak{\Xi}_{2 k-2}\left(\Gamma_{1}\right)$. Then there exist complex numbers $u_{ \pm}(f)$ uniquely determined up to $\mathbf{Q}(f)^{\times}$multiple such that $\frac{\Gamma(m) L(m, f, \chi)}{\tau(\chi)(2 \pi \sqrt{-1})^{m} u_{j}(f)} \in \mathbf{Q}(f)(\chi)$ for any integer $0<m \leq 2 k-3$ and a Dirichlet character $\chi$ such that $j=(-1)^{m} \chi(-1)$.

(2) Let $h$ be a Hecke eigenform in $\mathfrak{S}_{k-1 / 2}^{+}\left(\Gamma_{0}(4)\right)$ and $S(h)$ the normalized Hecke eigenform in $\mathfrak{\Xi}_{2 k-2}^{+}\left(\Gamma_{1}\right)$ corresponding to $h$ under the Shimura correspondence. Furthermore, for an integer $l$ such that $k>l \geq 2$, and a primitive character $\xi$ of conductor $M$, let $g$ be an element of $\mathfrak{P}_{l / 2-1}\left(\Gamma_{0}(4 M), \xi\right)$. Then $\frac{L(m / 2, h, g)}{u_{-}(S(h)) \pi^{m-1} \sqrt{-1}} \in$ $\mathbf{Q}(h) \mathbf{Q}(g)$ for any odd integer $m$ such that $1 \leq m \leq 2 k-3$ and a Dirichlet character $\eta$.

Now we state our main result in this paper.

Theorem 4.2. There exists a positive integer $r=r_{h}$ such that the values $L\left(l, h, \mathcal{E}_{3 / 2}^{\left(\chi_{1}\right)}\right), \ldots, L\left(l, h, \mathcal{E}_{3 / 2}^{\left(\chi_{r+1}\right)}\right)$ are linearly dependent over $\overline{\mathbf{Q}}$ for any integer $1 \leq l \leq k-2$ and Dirichlet characters $\chi_{1}, \ldots, \chi_{r+1}$ of odd conductors such that 
$\chi_{1}^{2}, \ldots, \chi_{r}^{2}, \chi_{r+1}^{2}$ are primitive. In particular, the values $L\left(l, h, \mathcal{E}_{3 / 2}^{\left(\chi_{1}\right)}\right), \ldots, L\left(l, h, \mathcal{E}_{3 / 2}^{\left(\chi_{r+1}\right)}\right)$ are linearly dependent over $\overline{\mathbf{Q}}$ for any non-quadratic characters $\chi_{1}, \ldots, \chi_{r+1}$ of odd prime conductors.

Proof. The assertion can be proved by Theorems 2.3 and Proposition 1.2.

Remark. We note that the Dirichlet series $L\left(s, h, \mathcal{E}_{3 / 2}^{\left(\chi_{1}\right)}\right), \ldots, L\left(s, h, \mathcal{E}_{3 / 2}^{\left(\chi_{r+1}\right)}\right)$ themselves are linearly independent over $\mathbf{C}$ in general. To show this, take a cuspidal Hecke eigenform

$$
h(z)=\sum_{m=1}^{\infty} c(m) \mathbf{e}(m z)
$$

in Kohnen's plus subspace of some weight $k-1 / 2$ for $\Gamma_{0}(4)$ such that $c(4) \neq 0$. Then there exist positive odd integers $n_{1}, n_{2}, \ldots, n_{r+1}$ coprime with each other such that $c\left(4 n_{i}^{2}\right) \neq 0$ for any $i=1, \ldots, r+1$. We also have $H_{1}\left(4 n_{i}^{2}\right) \neq 0$ for any $i=1, . ., r+1$. Then we can easily show that there exist Dirichlet characters $\chi_{1}, \ldots, \chi_{r+1}$ satisfying the conditions in Theorem 4.2 and

$$
\text { (*) } \operatorname{det}\left(\chi_{i}\left(4 n_{j}^{2}\right)\right)_{1 \leq i, j \leq r+1} \neq 0 .
$$

For an exampe, put $M_{i}=\prod_{1 \leq l \leq r+1, l \neq i} n_{l}^{2}$ for each $i$, and take a Dirichlet charcter mod $M_{i}$ such that $\chi_{i}^{2}$ is primitive. This is possible since we have $M_{i}>3$. Then these $\chi_{1}, \ldots, \chi_{r+1}$ satisfy the required properties. Now the $4 n_{j}^{2}$-th Fourier coefficient of $\mathcal{E}_{3 / 2}^{\left(\chi_{i}\right)}$ is

$$
\gamma_{i} H_{1}\left(4 n_{j}^{2}\right) \chi_{i}\left(4 n_{j}^{2}\right)
$$

with some non-zero number depending only on $i$, and therefore the $4 n_{j}^{2}$-th Dirichlet coefficient of $L\left(s, h, \mathcal{E}_{3 / 2}^{\left(\chi_{i}\right)}\right)$ is

$$
\gamma_{i} c\left(4 n_{j}^{2}\right) H_{1}\left(4 n_{j}^{2}\right) \chi_{i}\left(4 n_{j}^{2}\right) .
$$

Thus by $\left(^{*}\right), L\left(s, h, \mathcal{E}_{3 / 2}^{\left(\chi_{1}\right)}\right), \ldots, L\left(s, h, \mathcal{E}_{3 / 2}^{\left(\chi_{r+1}\right)}\right)$ are linearly independent over $\mathbf{C}$.

Theorem 4.3. Let $h$ be a Hecke eigenform in $\mathfrak{\Xi}_{k-1 / 2}^{+}\left(\Gamma_{0}(4)\right)$ and $S(h)$ be the normalized Hecke eigenform in $\mathfrak{S}_{2 k-2}^{+}\left(\Gamma_{1}\right)$ corresponding to $h$ under the Shimura correspondence. Assume that all the Fourier coefficients of h belong to $\mathbf{Q}(S(h))$. Let $\chi$ be a Dirichlet character of conductor $N$. Assume that $\chi^{2}$ is primitive, and that $p \equiv-1 \bmod 4$ for some prime factor $p$ of $N$. Then for any odd integer $m$ such that $1 \leq m \leq 2 k-3$, the value $L\left(m / 2, M(h), \chi^{2}\right)$ belongs to the vector space $\mathbf{Q}(S(h))(\chi) u_{-}(S(h)) \pi^{m-1} \sqrt{-1}$.

Proof. By assumption, $\mathcal{E}_{3 / 2}^{(\chi)}$ belongs to $\mathfrak{R}_{3 / 2}\left(\Gamma_{0}\left(4 N^{2}\right), \chi^{2}\right)$ whose Fourier coefficients belong to $\mathbf{Q}(\chi)$. Thus the assertion can be proved by Theorem 2.3 and (1) of Proposition 4.1. 
Remark. It is expected that (2) of Proposition 4.1 remains valid for $g=E_{3 / 2}^{(\eta)}$. Thus it is expected that Theorem 4.3 remains valid without the assumption on the congruence of a prime factor of $N$. As remarked in the Introduction, the above theorem is not a special case of Choie and Kohnen's result. Actually they treated the values of Koecher-Maaß series at integers. On the other hand, we treat the values at half-integers. This type of result has not been given in case $n=1$, and is not expected in case $n$ is odd. It seems interesting to know whether this type of algebraicity holds for the special values at half-integer of twisted Koecher-Maaß series of any cusp form of even degree.

Finally by the functional equation in Proposition 1.1, we obtain:

Theorem 4.4. Put

$$
\Lambda\left(s, h, \mathcal{E}_{3 / 2}^{(\chi)}\right)=N^{2 s} \pi^{-2 s} \tau\left(\chi^{2}\right)^{-1} L\left(s, h, \mathcal{E}_{3 / 2}^{(\chi)}\right) .
$$

Assume that $\chi^{2}$ is primitive. Then $\Lambda\left(s, h, \mathcal{E}_{3 / 2}^{(\chi)}\right)$ has an analytic continuation to the whole s-plane and has the following functional equation:

$$
\Lambda\left(k-s, h, \mathcal{E}_{3 / 2}^{(\chi)}\right)=\Lambda\left(s, h, \mathcal{E}_{3 / 2}^{(\bar{\chi})}\right) .
$$

The meromorphy of this type of series was derived in [12] by using so called the Rankin-Selberg integral expression in more general setting, but we don't know whehter the functional equation of the above type can be directly proved without using the above method.

\section{References}

[1] T. Arakawa, Math. Ann., 238(1978), 157-173,

[2] S. Böcherer, Bemerkungen über die Dirichletreichen von Koecher und Maaß, Math. Gottingensis des Schrift. des SFB. Geometry and Analysis Heft 68(1986).

[3] Y. Choie and W. Kohnen, Special values of Koecher-Maaß series of Siegel cusp forms, Pacific J. Math. 198 (2001), 373-383.

[4] T. Ibukiyama and H. Katsurada, An explicit formula for Koecher-Maaß Dirichlet series for Eisenstein series of Klingen type, J. Number Theory, 102(2003), 223-256.

[5] M. Eichler and D. Zagier, The theory of Jacobi forms, Progress in Math., vol. 55, Birkhäuser Boston Inc., Boston, Mass. 1985. 
[6] T. Ibukiyama and H. Katsurada, An explicit formula for Koecher-Maaß Dirichlet series for Eisenstein series of Klingen type, J. Number Theory, 102(2003), 223-256.

[7] T. Ibukiyama and H. Katsurada, An explicit formula for Koecher-Maaß Dirichlet series for the Ikeda lifting, Abh. Math. Sem. Hamburg 74(2004), 101-121

[8] W. Kohnen, W. Kohnen, Newforms of half-integral weight, J. reine und angew. Math. 333(1982) 32-72.

[9] H. Maaß, Siegel's modular forms and Dirichlet series, Lecture Notes in Math., vol. 216, Berlin-Heidelberg-New York Springer 1971.

[10] Y. Mizuno, The Rankin-Selberg convolution for Cohen's Eisenstein series of half-integral weights, Abh. Math. Sem. Hamburg 75(2005),1-20.

[11] G. Shimura, On the periods of modular forms, Math. Ann. 229(1977), 211221.

[12] G. Shimura, The critical values of certain zeta functions associated with modular forms of half-integral weight, J. Math. Soc. Japan 33(1981), 649672 .

Hidenori Katsurada

Muroran Institute of Technology

27-1 Mizumoto, Muroran

050-8585 Japan

E-mail: hidenori@mmm. muroran-it.ac.jp

Yoshinori Mizuno

Faculty and School of Engineering

The University of Tokushima

2-1, Minami-josanjima-cho Tokushima

770-8506, Japan

E-mail:mizuno@pm.tokushima-u.ac.jp 